\title{
A Technique for Detection and Analysis of Human Breast Cancer
}

\author{
Prof. Samir Kumar Bandyopadhyay \\ Professor, Dept. of Computer Science \& Engineering, \\ University of Calcutta, \\ 92 A.P.C. Road, \\ Kolkata - 700009, India.
}

\begin{abstract}
Imaging techniques can play an important role in helping perform breast biopsies, especially of abnormal areas. In this paper we try to identify cancer parts just using simple technique of isolation of insignificant portion of slide by color polarization. Here an attempt is made to analyze the biopsy slides for identification and detection of the area of affected cancer. In the era computer and telecommunications, pathologist's still mount tissue slices on glass slides, treat them with appropriate stains and examine them through a microscope. Despite advances in staining techniques, it's a process that has changed little over the last twenty years. Interpreting requires a great deal of skill and experience and it is also time consuming.
\end{abstract}

\section{Keywords}

Human Breast Cancer, FNAC, Mammography, MRI, Surgical biopsy, Bi-color monochrome image and Inverse color.

\section{INTRODUCTION}

Imaging techniques play an important role in helping perform breast biopsies, especially of abnormal areas that cannot be felt but can be seen on a conventional mammogram or with ultrasound. One type of needle biopsy, the stereotactic-guided biopsy, involves the precise location of the abnormal area in three dimensions using conventional mammography. Stereotactic refers to the use of a computer and scanning devices to create three-dimensional images [3].

Another type of needle biopsy uses a different system, known as the Mammotome breast biopsy system. A large needle is inserted into the suspicious area using ultrasound or stereotactic guidance. The Mammotome is then used to gently vacuum tissue from the suspicious area. Additional tissue samples can be obtained by rotating the needle.

Digital mammography is a technique for recording $\mathrm{x}$-ray images in computer code instead of on x-ray film, as with conventional mammography. The images are displayed on a computer monitor and can be enhanced (lightened or darkened) before they are printed on film. Images can also be manipulated; the radiologist can magnify or zoom in on an area. From the patient's perspective, the procedure for a mammogram with a digital system is the same as for conventional mammography [3].

Digital mammography may have some advantages over conventional mammography. The images can be stored and retrieved electronically, which makes long-distance consultations

\author{
Indra Kanta Maitra \\ Research Fellow, Dept. of Computer Science \& Engg, \\ University of Calcutta, \\ 92 A.P.C. Road, \\ Kolkata - 700009, India.
}

with other mammography specialists easier. Because the radiologist can adjust the images, subtle differences between tissues may be noted. Despite these benefits, studies have not yet shown that digital mammography is more effective in finding cancer than conventional mammography.

The first digital mammography [2] system received U.S. Food and Drug Administration (FDA) approval in 2000. An example of a digital mammography system is the Senographe 2000D. Many more techniques are available other than the cytogenetic processes, however, these are imaging technologies to detect, diagnose, and characterize breast.

Most types of cancer cells eventually form a lump or mass called a tumor, and are named after the part of the body where the tumor originates. Breast cancer begins in breast tissue, which is made up of glands for milk production, called lobules, and the ducts that connect lobules to the nipple. The remainder of the breast is made up of fatty, connective and lymphatic tissue.

a) Most masses are started to show they are not cancerous, do not grow uncontrollably or spread, and are not life threatening.

b) Some breast cancers are called in situ because they are confined within the ducts (ductal carcinoma in situ) or lobules (lobular carcinoma in situ) of the breast. Nearly all cancers at this stage can be cured. Many oncologists believe that lobular carcinoma in situ (also known as lobular neoplasia) is not a true cancer, but an indicator of increased risk for developing invasive cancer in either breast.

c) Most cancerous breast tumours are invasive, or infiltrating. These cancers start in the lobules or ducts of the breast but have broken through the duct or glandular walls to invade the surrounding tissue of the breast.

The seriousness of invasive breast cancer is strongly influenced by the stage of the disease; when it is first detected [1].

\section{REVIEW WORKS}

We are discussing some review works, which are very recent and related to our paper.

V. Mallapragada et al, October 2007, presented [4,5] a new concept for real-time manipulation of a tumor using a robotic force controller that monitored the image of the tumor to generate appropriate force to position the tumor at a desired location. The idea behind concept was possible to manipulate a tumor in real- 
time by applying controlled external force in an automated way. The success of this approach had the potential to reduce the number of attempts a surgeon make to capture the desired tissue specimen, minimized tissue damage, improved speed of biopsy, and reduced patient discomfort.

Cigdem Gunduz et al, 2004, reported a computational method that modeled a type of brain cancer using topological properties of cells in the tissue image. They constructed the graphs based on the locations of cells within the image. They used the Waxman model in their experiment [6].

C. Cagatay Bilgin et al, 2007, classified [7] the breast cancer tissues using graph theory. Image segmentation approach was used and Euclidean Distances were calculated between vertices [7]. Considering the cell locations generated cell Graphs. These approaches toward automatic detection of cancer were actually failed because the types of cancers identified more and more.

A.M. Tang et al, 2008, proposed, simultaneous capturing of ultrasound (US) and magnetic resonance (MR) images allowed fusion of information obtained from both modalities. An MRcompatible US system where MR images were acquired in a known orientation with respect to the US imaging plane and concurrent real-time imaging could be achieved. Compatibility of the two imaging devices was a major issue in the physical setup. Tests were performed to quantify the radio frequency (RF) noise introduced in MR and US images, with the US system used in conjunction with MRI scanner of different field strengths $(0.5 \mathrm{~T}$ and 3T). Their results indicated that simultaneous US and MR imaging were feasible with properly designed shielding, resulting in negligible broadband noise and minimal periodic RF noise in both modalities. US could be used for real time display of the needle trajectory, while MRI could be used to confirm needle placement [8].

C. Zhu, et al, 2009, have explored [9] the use of a fiber-optic probe for in vivo fluorescence spectroscopy of breast tissues during percutaneous image-guided breast biopsy. A total of 121 biopsy samples with accompanying histological diagnosis were obtained clinically and investigated in their study. The tissue spectra were analyzed using partial least-squares analysis and represented using a set of principal components (PCs) with dramatically reduced data dimension. For nonmalignant tissue samples, a set of PCs that account for the largest amount of variance in the spectra displayed correlation with the percent tissue composition. This study demonstrated the feasibility of performing fluorescence spectroscopy during clinical core needle breast biopsy, and the potential of that technique for identifying breast malignancy in vivo.

Lin Yang, et al, 2007, introduced a Grid-enabled CAD to perform automatic analysis of imaged histopathology breast tissue specimens [10]. More than 100,000 digitized samples $(1200 \times 1200$ pixels $)$ were processed on the Grid. They analyzed results for 3744 breast tissue samples, which were originated from four different institutions using diaminobenzidine (DAB) and hematoxylin staining. Both linear and nonlinear dimension reduction techniques were compared, and the best one was applied to reduce the dimensionality of the features. The results shown that the Gentle Boosting using an eight node CART decision tree as the weak learner provided the best result for classification. The algorithm has an accuracy of $86.02 \%$ using only $20 \%$ of the specimens as the training set.

\section{PROPOSED METHOD}

In our work, first we try to understand the type of human breast cancer and attempt to analyze the histopathological slides with our proposed method to identify cancer parts.

LCIS (Lobular Carcinoma in Situ) - Lobular cancer in situ (LCIS) means that there are cell changes inside the breast lobes. This is not cancer. A patient having LCIS means that you have an increased risk of getting breast cancer in the future.

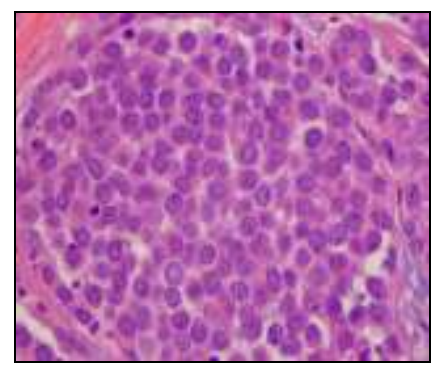

Figure 1. Lobular Carcinoma in Situ

DCIS (Ductal Carcinoma in Situ) - If you have ductal cancer in situ (DCIS), it means that cells inside some of the ducts of your breast have started to turn into cancer cells. These cells are all inside the ducts and have not started to spread into the surrounding breast tissue. So, there is very little chance that any of the cells have spread to the lymph nodes or elsewhere in the body.

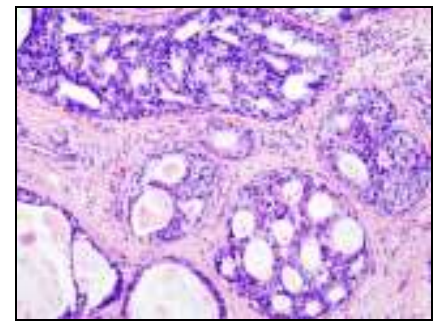

Figure 2. Ductal Carcinoma in Situ Invasive

Ductal Breast Cancer is the most common type of breast cancer. Between 70 and 80 out of every 100 breast cancers diagnosed (70 - $80 \%$ ) are this type. A ductal carcinoma of the breast is a cancer that is growing in the cells that line the ducts of the breasts.

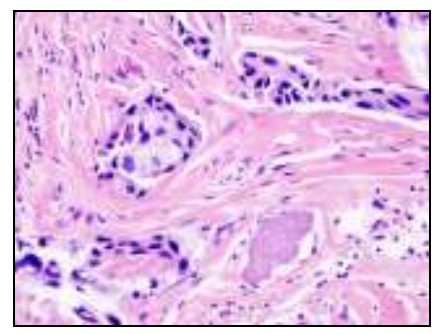

Figure 3. Invasive Ductal Breast Cancer 
Invasive Lobular Breast Cancer - About 1 in 10 breast cancers diagnosed $(10 \%)$ are invasive lobular carcinoma. Invasive lobular cancer is most common in women between 45 and 55 years old. It is possible for men to get invasive lobular breast cancer, but this is very rare.

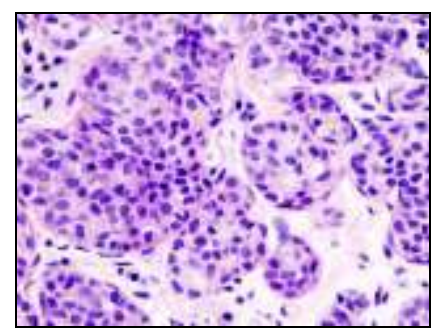

Figure 4. Invasive Lobular Breast Cancer

\subsection{Identification and Detection}

We have to understand the structure of normal breast and identify the abnormality due to propagation of cancer.

The breasts are made up of fat, connective tissue, and gland tissue divided into lobes. A network of ducts spreads from the lobes towards the nipple. Younger women have more glandular tissue in their breasts, which makes them dense. Once a woman is past her menopause, the glandular tissue is gradually replaced by fat, which is less dense. A 'tail' of breast tissue leads into the armpit (axilla). The armpits have many lymph glands, also known as lymph nodes. They are part of the lymphatic system. The lymphatic system is made up of a network of lymph glands, connected throughout the body by tiny tubes called lymph vessels.Lymph glands are serious in cancer care because the lymph to the nearest lymph glands can carry any cancer cells that have broken away from a tumour. If a patient has cancer, but no cancer cells in any of your lymph glands, then it will less likely to have spread.

a) Mammography is a low-dose x-ray procedure that allows visualization of the internal structure of the breast. Mammography is highly accurate, but like most medical tests, it is not perfect. On average, mammography will detect about $80 \%-90 \%$ of the breast cancers in women without symptoms. Testing is somewhat more accurate in postmenopausal than in premenopausal women [12]. The small percentage of breast cancers that are not identified by mammography may be missed for just as mammography uses x-ray machines designed especially to image the breasts, breast MRI also requires special equipment. Higher-quality images are produced by dedicated breast MRI equipment than by machines designed for head, chest, or abdominal MRI scanning. However, many hospitals and imaging centres do not have dedicated breast MRI equipment available. It is important that screening MRI's are done at facilities that are capable of performing an MRI-guided breast biopsy at the time of the exam if abnormalities are found. Otherwise, the scan must be repeated at another facility at the time of the biopsy.

Initial mammographic or MRI images themselves are not usually enough to determine the existence of a benign or malignant disease with certainty. If a finding or spot seems suspicious, your radiologist may recommend further diagnostic studies. Interpretations of mammograms can be difficult because a normal breast can appear differently for each woman. Also, the appearance of an image may be compromised if there is powder or salve on the breasts or if you have undergone breast surgery. Because some breast cancers are hard to visualize, a radiologist may want to compare the image to views from previous examinations. Not all cancers of the breast can be seen on mammography [14].

b) Fine Needle Aspiration Cytology (FNAC) is a method of collecting samples for cytological examination of a solid tissue or tumour with the help of a fine needle. This is an easy and simple method to collect the specimen. No anesthesia is required and no special preparation is required. It has high degree of sensitivity and specificity in the diagnosis is of most of the lesions. It is used in highly used in breast cancer today [13]. Most of the studies have confirmed correct results in about $96 \%$ of the tests, which is very significant. It is only used in solid tumours of breast tissue. In radiologically positive cases of carcinoma breast, FNAC sensitivity is $61 \%$ and core biopsy sensitivity is $97 \%$. In radiologically undiagnosed cases, FNAC sensitivity is $53 \%$ but core biopsy sensitivity is $96 \%$. Automated core needle biopsy has superior diagnostic power than FNAC.

c) A core biopsy is a procedure where a needle is passed through the skin to take a sample of tissue from a mass or lump. The tissue is then examined under a microscope for any abnormalities. Core biopsy may be performed when a suspicious lump is found, for example a breast lump or enlarged lymph node, or if an abnormality is detected on an imaging test such as x-ray, ultrasound or mammography.

d) Core biopsy is a more invasive procedure than fine needle aspiration biopsy; however, it is quicker and less invasive than a surgical biopsy. In some cases, the result of a core biopsy will prevent the need for surgery to take place. If you have a breast lump and want it checked out, a surgical biopsy is a good way to get a clear diagnosis. This type of breast biopsy removes the largest size of tissue sample, as compared to any type of needle biopsy. In some cases, the entire mass and a margin of healthy tissue may be removed. The tissue will be examined in a pathological lab right away to ensure that it is an accurate sample and get a diagnosis. Surgical breast biopsy takes the largest tissue sample and has the highest accuracy rate of all biopsy methods.

A pathology lab can use two methods to study your tissue sample. The quickest method is called "frozen section" or cryosection. The tissue is rapidly frozen and sliced with a special blade into a section thin enough to see through. A permanent section method is a more thorough process, using special chemicals to get more information from the tissue slide [15].

Digital detection process comes in the picture at that moment. The image of histopathological slide under microscope can be processed through digital image processing to detect cancer 
accurately. Now it is the question that what is the need of digital detection technique where it can be detect easily by human eye itself. Answer is very simple; in all the cases human-error is one of most important factor, which cannot be eliminated completely, especially when it is detection of cancer, which can cause death of the patient. However, there are many more additional advantages of digital detection method:

a) Distribution and sharing of the digitally processed images of histopathological slide to remote location is much more easily and less time consuming than send the original one to the experts for opinion.

b) Preservation of digitally processed images of histopathological slide is much simple for future references. In a study, the Department of Pathology at the University Medical Centre (UMC) Utrecht processes some 300 to 500 histopathological slides per day, nearly 100,000 annually, each of which must be processed, scored, and importantly stored; if patients return to the hospital, their slides may need to be re-examined. Oftentimes, though, that isn't so simple. Glass slides can break or deteriorate over time. They can become lost; Utrecht's archive holds some 7 million slides weighing $60,000 \mathrm{~kg}$, and its 15 pathologists and 10 or so residents add another $600 \mathrm{~kg}$ annually. Further most significantly, a slide can be under the lens of only one microscope at a time, creating logistical problems for consultations, conferences, and training sessions, and meaning, by extension, that if you mail off a slide for a second opinion, it's gone until the colleague returns it [17].

c) The increased demand is not currently being matched by the supply of skilled pathologists, who remain in short supply in most parts of the world. Therefore, a real need for digital technology that both improves the efficiency of pathology labs, reduce investigation time and helps pathologists with their diagnoses [18].

d) In pathological investigation chemical reagents are used which are highly toxic, costly and dangerous, the said process will be reduce by using digital image processing. So, it is not only effective and less costly but also ecofriendly too.

e) Destruction and recycling of these highly toxic slides is also very costly and hazardous, but in the said process it is absolutely not required.

f) Decrease the human-error under microscopic investigation of histopathological slide.

g) Reduce investigation cost drastically.

h) Last but not list it is not sublimating the traditional process but it is providing aid to it for further accuracy and improvement.

\subsection{Digital Detection Method}

In our work, used free Tissue Blocks downloaded from OriGene Technologies, Inc, 2009 [16]. In the experiments, different breast cancer tissues from different patients and different non-cancerous falsely detected breast tissues from different normal females are considered. Each of the 24-bit bitmap image size is $640 \mathrm{X} 480$ Pixels.

Step 1. Convert the 24-bit color bitmap image to 256-shaded grey scale image after increasing contrast of the source image.

Step 2. Convert the resultant 256-shaded grey scale image to Inverse $\mathrm{Bi}$-Color Monochrome image after increasing contrast of the source image.

\section{TEST RESULT}

Major objective of the algorithms, to remove the huge amount of fat, connective tissue, and gland tissue from the Cancerous cells within the histopathological biopsy samples. The stage, intensity, type, future and treatment of cancer can only be detected on the basis of orientation of malignant cell, shape of the cell and duct, density carcinogenic cells in compare with normal cells. The outputs of aforesaid algorithms are depicted in the following figure for cancerous cells within the biopsy slide and normal slide along with the histogram of the images.

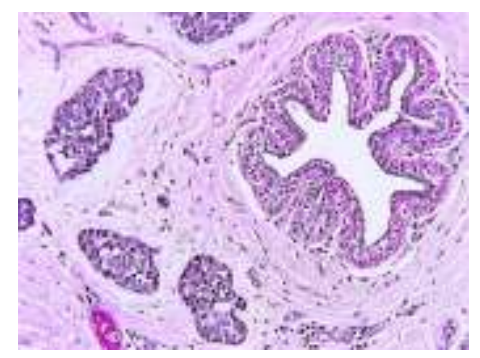

Figure 5. 24-bit Color Image of Histopathological Slide showing Cancer

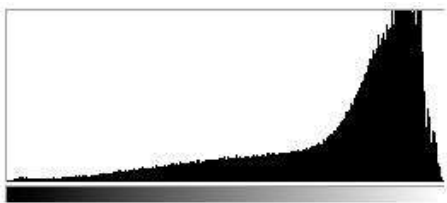

Figure 6. Histogram of figure 5

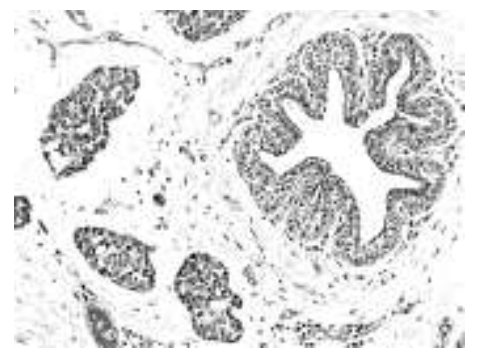

Figure 7. 256-shade Grey Image of figure 5

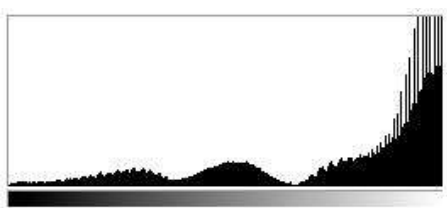

Figure 8 . Histogram of figure 7 


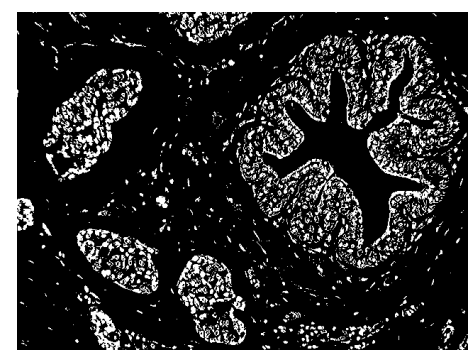

Figure 9. Inverse Bi-Color Image of figure 5

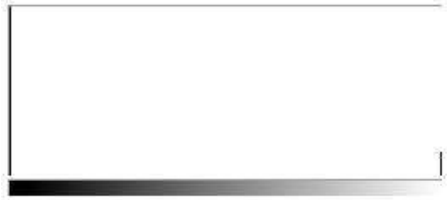

Figure 10. Histogram of figure 9

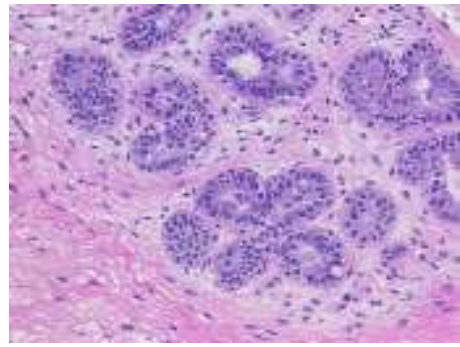

Figure 11. 24-bit Color Image of Histopathological Slide showing Normal Tissue

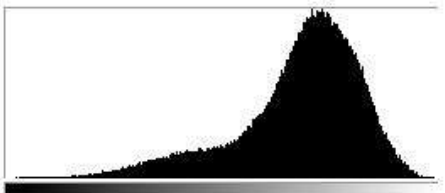

Figure 12. Histogram of figure 11

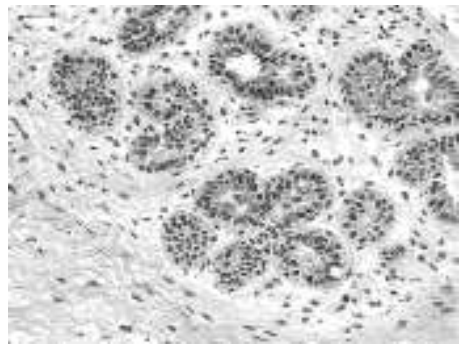

Figure 13. 256-shade Grey Image of figure 11

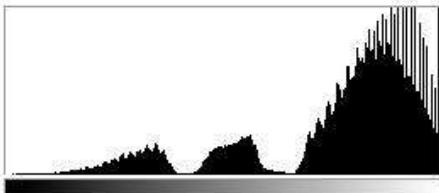

Figure 14. Histogram of figure 12

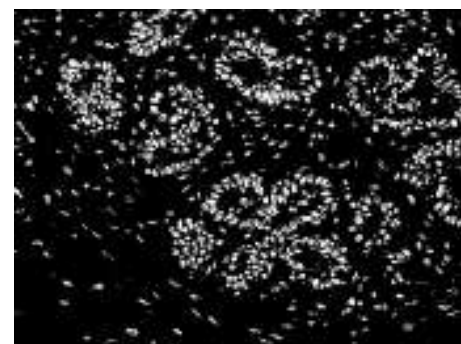

Figure 15. Inverse Bi-Color Image of figure 11

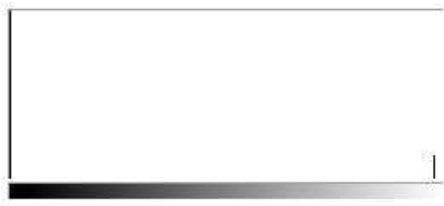

Figure 16. Histogram of figure 15

Figure 5, 7and 9 shows the Cancerous tissue. In figure 9 all the fat, connective tissue, and gland tissue are isolated. It is only showing the parts, which are important to determine the carcinoma. It is depicting the abnormal orientation of malignant cell, shape of the cell and duct. In contrast, figure 11, 13 and 15 shows the normal slide and all the fat, connective tissue, and gland tissue are isolated in figure 15. The orientation, shapes etc. in figure are normal in nature and showing no sign of carcinoma.

The image histograms are showing the color clustering on the basis of algorithms. In view of the major objective to isolate less significant parts from the considerable portion of slide, firstly we have increased the contrast of the color image for prominence and polarization of colors, which is followed by converting the image to grey scale. The polarization of color is highly proved by the aforesaid histograms provided. Clustering of grey color is easier than RGB image. Again we have increased the contrast grey image to do the prominence and polarization of color further, which reduced the color spectrum drastically, and help to isolate one portion of image from other appreciably. Later converting the image to inverse bi-color monochromic image totally eliminates the insignificant part of the slide.

\section{CONCLUSION}

It can be very difficult to decide who may have a breast cancer and who may have a non-cancerous breast condition. Advances in computing and telecommunications have resulted in the availability of a range of tools for use in pathology quality assurance and support system. The majority focuses on either enabling pathologists to examine and diagnose cases, or providing image archives that serve as reference material. Limited emphasis has been placed on analyzing the diagnostic process used by pathologists to reach a diagnosis and using this as a resource for improving diagnostic performance. This method has potential for further development because of its simplicity that will motivate online or real-time breast cancer diagnosis in providing the opinion. 


\section{REFERENCES}

[1] Breast Cancer Facts \& Figures, 2009-2010, American Cancer Society, Inc.

[2] FDA Web site, http://www.accessdata.fda.gov/scripts/ cdrh/cfdocs/cfMQSA/mqsa.c fm

[3] National Cancer Institute (NCI) Web site, http://www.cancernet.gov

[4] V. Mallapragada,N.Sarkar and T.K. Podder, "A Robotic System for Real-time Tumor Manipulation During Image guided Breast Biopsy", IEEE International Conference on Bioinformatics and Bioengineering, October 14-17, 2007, Boston, MA, pp. 204-210.

[5] V. Mallapragada, N.Sarkar, and T.K. Podder, "RobotAssisted Real-Time Tumor Manipulation for Breast Biopsy", IEEE Transactions on Robotics, Vol. 25, Issue 2, April 2009, pp. 316-324.

[6] Cigdem Gunduz, Bulent Yener and S. Humayun Gultekin, "The cell graphs of cancer", Bioinformatics, Oxford University Press, Vol 20, Issue 1, January, 2004, pp. 145151.

[7] C. Cagatay Bilgin, Cigdem Demir, Chandandeep Nagi, and Bulent Yener, "Cell-Graph Mining for Breast Tissue Modelling and Classification", 29th IEEE EMBS Annual International Conference, August 23-26, 2007, Lyon, France.

[8] A.M. Tang, D.F. Kacher,E.Y.Lam, K.K.Wong, F.A. Jolesz, and E.S. Yang, "Simultaneous Ultrasound and MRI System for Breast Biopsy: Compatibility Assessment and Demonstration in a Dual Modality Phantom", IEEE Transactions on Medical Imaging, Vol. 27, Issue 2, February 2008, Davis, CA, USA, pp. 247-254.
[9] C. Zhu, E.S. Burnside, G.A. Sisney, L.R.Salkowski, J.M. Harter, B. Yu, and N. Ramanujam, "Fluorescence Spectroscopy: An Adjunct Diagnostic Tool to Image- Guided Core Needle Biopsy of the Breast", IEEE Transactions on Biomedical Engineering, Vol. 56, Issue 10, October 2009, pp. $2518-2528$

[10] Lin Yang, Wenjin Chen, Peter Meer, Gratian Salaru, Michael D. Feldman, and David J. Foran, "High Throughput Analysis of Breast Cancer Specimens on the Grid", MICCAI 2007, Part I, October 29 - November 2, 2007, Brisbane, Australia, LNCS 4791/2007, pp. 617-625

[11] About Breast Cancer - A Quick Guide, Cancer Research UK's Patient Information Website Cancer Help UK, July 2009

[12] Michaelson J, Satija S, Moore R, et al. The pattern of breast cancer screening utilization and its consequences. Cancer. Jan 1 2002, 94(1), pp 37-43

[13] Fine Needle Aspiration Cytology (F.N.A.C.) INV-34 Shuja Tahir, FRCSEd Riaz A Bhutta

[14] RadiologyInfo.org developed jointly by Radiological Society of North America and American College of Radiology.

[15] About.com: Breast Cancer. Open Surgical Breast Biopsy By Pam Stephan, About.com Guide Updated August 13, 2008, Health's Disease and Condition content is reviewed by the Medical Review Board.

[16] Origene Web site, http://www.origene.com 